\title{
Risk of immune-related colitis with PD-1/PD-L1 inhibitors vs chemotherapy in solid tumors: systems
} \section{assessment}

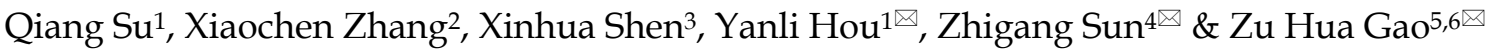 \\ 1. Department of Oncology, Beijing Friendship Hospital, Capital Medical University, Beijing, 100050, China; Department of Pathology, Research Institute of \\ McGill University Health Center, Montreal, Quebec, Canada \\ 2. Department of Biomedical Engineering, School of Medicine, Tsinghua University, Beijing 100084, China \\ 3. Department of Biomedical Engineering of Anatomy, Histology and Embryology, Institute of Basic Medical Sciences Chinese Academy of Medical Sciences, \\ School of Basic Medicine Peking Union Medical College \\ 4. Department of Thoracic Surgery, Jinan Center Hospital Affiliated to Shandong University, Shandong University, Jinan, 250014, China \\ 5. Department of Pathology, Research Institute of McGill University Health Center, Montreal, Quebec, Canada \\ 6. Liaocheng City Hospital, Shandong Province, PR China \\ $\bowtie$ Corresponding authors: Y.L.H., Z.G.S. and Z.H.G (Tel +86-13146303355; email: hyl_0730@126.com, sunzhigangzm@126.com, and zu-hua.gao@mcgill.ca)
}

(c) Ivyspring International Publisher. This is an open access article distributed under the terms of the Creative Commons Attribution (CC BY-NC) license (https://creativecommons.org/licenses/by-nc/4.0/). See http://ivyspring.com/terms for full terms and conditions.

Received: 2017.12.05; Accepted: 2018.01.27; Published: 2018.04.12

\begin{abstract}
Background: We performed a meta-analysis to evaluate the risk of immune-related colitis associated with PDI/PD-L1 inhibitors as compared to chemotherapy in solid tumor patients.

Methods: Eligible studies were identified through a comprehensive search of multiple databases and included solid tumor patients in randomized controlled trials (RCTs) with PD-1/PD-L1 inhibitors. The data was analyzed by Stata version 12.0 software.

Results: After exclusion of ineligible studies, 11 clinical trials were considered eligible for the meta-analysis, including 5751 patients. Compared with chemotherapy, the risk ratios (RRs) of all-grade colitis were significant for the PD-1 inhibitor subgroup (RR 2.69, 95\% confidence interval $(\mathrm{Cl})$ : 1.15-6.29, $\mathrm{p}=0.023)$, and for pembrolizumab subgroup ( $R R$ 3.17, $95 \% \mathrm{Cl}: 1.08-9.37, \mathrm{p}=0.037)$, but not for nivolumab treatment and PD-L1 inhibitor (atezolizumab) treatment (RR 2.05, 95\% Cl: 0.52-8.13, $p=0.305$; RR 4.75,95\% Cl: 0.56-40.50, $p=0.154$, respectively). The $R R$ of all-grade colitis was significant for PD-1/PD-L1 inhibitor in NSCLC (RR 4.34, 95\% Cl: 1.37-13.82, $p=0.013$ ), and not significant in melanoma (RR 2.11, 95\% Cl: 0.54-8.34, $p=0.285$ ). Moreover, the RRs of all-grade diarrhea were significant for the PD-1 inhibitor subgroup (RR 0.61, 95\% Cl: $0.44-0.83, p=0.002$ ), for the nivolumab subgroup ( $R R 0.54,95 \% \mathrm{Cl}: 0.34-0.87, p=0.012$ ), and for atezolizumab subgroup ( $R R$ $0.48,95 \% \mathrm{Cl}: 0.25-0.89, \mathrm{p}=0.021)$. The RR of high-grade diarrhea was significant for atezolizumab subgroup (RR 0.34, 95\% Cl: 0.12-0.94, $\mathrm{p}=0.037$ ).

Conclusions: Our meta-analysis demonstrates that compared with chemotherapy, pembrolizumab may result in a higher risk of all-grade immune-mediated colitis. PD-1/PD-L1 inhibitor treatment in NSCLC patients, but not in melanoma patients, increases the risk of all-grade colitis incidence.
\end{abstract}

Key words: Immune-related colitis; PD-1; PD-L1; Cancer; Meta-analysis

\section{Introduction}

Cancer is one of the most serious and costly health problems in the USA. About 1.68 million new cancer cases and 60 thousand new cancer deaths are estimated to occur in 2017. [1]. Targeted therapy of immune checkpoints including PD-1 (programmed cell death protein 1), and PD-L1 (programmed cell death ligand 1) is a breakthrough in advanced cancer therapy that can overcome immune tolerance to 
cancer cells [2]. Clinically, as more PD-1/PD-L1 inhibitors have been used in anticancer therapy, more adverse events have been found. The adverse events of PD-1/PD-L1 inhibitors are due to a disruption in the immune balance in tissues. Immune checkpoint inhibitors generate some special toxicity profiles called immune-related adverse events (irAE) that include colitis and endocrine dysfunction [3]. Immune-related colitis after PD-1/PD-L1 inhibitors therapy has been reported in some RCTs, and its morbidity ranges from $0.3 \%$ to $2.3 \%$ for different PD-1/PD-L1 inhibitors observed in cancer patients applying those immune checkpoint inhibitors (ICIs) [4-14]. One study reported that immune-related colitis induced by ICIs was practically accompanied by diarrhea [15] and that severe colitis was even fatal. Therefore, awareness of the incidence and characteristics of immune-related colitis may guide the appropriate utilization of PD-1/PD-L1 inhibitors in clinical practice. This meta-analysis was conducted to determine the risk of immune-related colitis and diarrhea in cancer patients who have been treated with PD-1/PD-L1 inhibitor alone compared with chemotherapy.

\section{Methods}

A systematic review and meta-analysis was conducted according to the guidelines from the Cochrane Handbook for Systematic Reviews of Interventions [16], with reporting following the PRISMA Statement [17].

\section{Search strategy}

We searched the following databases: the Embase, and PubMed (up to November 20, 2017) for studies reporting the risk of immune-related colitis in PD-1/PD-L1 inhibitor therapies versus chemotherapy (Supplementary Table S1).

\section{Inclusion criteria}

Studies in English literature with the following information were included in our meta-analysis: (1) Phase II/III RCTs with primary endpoints such as overall survival (OS), progression-free survival (PFS), or objective response rate (ORR); (2) histologicallyconfirmed solid cancer including melanoma (MM), lung cancer, and others; (3) information on PD-1/PD-L1 inhibitors and chemotherapy, and colitis or diarrhea; and (4) some similarity in experimental methods across different studies.

\section{Data extraction}

Two independent reviewers (Q.S. and Y.L.H.) searched all the relevant studies and independently read the titles, abstracts, and full texts of the identified studies, and access a study considered to be appropriate for meta-analysis evaluated by using a patient, intervention, comparison and outcome (PICO) chart [18]. The following information was extracted by two independent reviewers (Q.S. and Y.L.H.) from the selected studies: year of publication, name of journal, author's last name, the primary endpoint, type of solid tumor, number of patients in the PD-1/PD-L1 inhibitor treatment and control groups, number of patients bearing colitis or diarrhea of all-grade (grade 1-5) and high-grade (grade 3-5) (Supplementary Table S2).

\section{Data analysis}

In our meta-analysis, the risk of bias analysis of our included studies was performed using Review Manager 5.3 software (Cochrane Collaboration 2014, Nordic Cochrane Center, Copenhagen, Denmark). Two reviewers (Q.S. and Y.L.H.) independently assessed the quality of the included RCTs according to the Cochrane risk of bias tool, which assesses the following seven domains: selection bias (including both random sequence generation and allocation concealment), performance bias, detection bias, attrition bias, reporting bias, and other bias. The Stata version 12.0 statistical software (Stata Corporation, College Station, Texas, USA) was used for the meta-analysis. The Risk ratio (RR) was used to estimate colitis and diarrhea (grade 1-5 and grade $3-5)$. The RR $>1.0$ indicates higher risk or higher incidence of colitis or diarrhea in patients treated with PD-1/PD-L1 inhibitor than chemotherapy treatment. In addition, the $\mathrm{Q}$ test and $\mathrm{I}^{2}$ statistics were used to assess the heterogeneity among the RCTs. I ${ }^{2}$ values of $<30 \%, 30 \%-59 \%, 60 \%-75 \%$, and $>75 \%$ were classified as low, moderate, substantial, and considerable heterogeneity, respectively [19]. We used the random-effects model (REM) described by DerSimonian and Laird [20] to calculate pooled RR and $95 \%$ confidence interval (CI). Sensitivity analysis was used by removing one study at a time, to examine whether the results could have been influenced by a single study, especially with a dubious result or considerable heterogeneity. Sources of heterogeneity were explored using subgroup analyses according to both different PD-1/PD-L1 inhibitors and different type of cancers. The Begg's and Egger's tests were used to analyze the publication bias. All $\mathrm{P}$ values were 2 -tailed, and a probability level $<0.05$ was considered statistically significant.

\section{Quality Assessment}

Firstly, two independent reviewers (Y.L.H. and Q.S.) searched all the relevant studies, and assessed studies appropriate for meta-analysis based on the 
evaluation using PICO chart, and assessed the risk of bias for the included studies by the Cochrane Handbook. All disagreements were resolved by discussion with the third reviewer (X.C.Z.) until a consensus was reached. Secondly, our analysis was performed by pair-wise comparisons of the PD-1/PD-L1 inhibitor arms with the chemotherapy arms. Among the studies, there were two three-arm trials. To avoid an increased influence of these trials on the overall result, the number of patients in the arm, which was used twice, was divided by two. Thirdly, we paid much attention to the heterogeneity among the RCTs by using sensitivity analysis and subgroup analysis. On the other hand, although the $\mathrm{I}^{2}$ value was $<30 \%$, instead of the fixed-effects model (FEM), REM was employed for our meta-analysis to verify the statistical results.

\section{Results}

\section{Selection of studies}

Using the search terminology, we initially identified 1331 studies from our database search. Among those 1331 studies, 11 RCTs met our strict inclusion criteria (Supplementary figure 1). All the included trials evaluated and compared the effectiveness of PD-1/PD-L1inhibitor therapies with chemotherapy in solid tumors, representing data from a total of 5751 patients (Nivolumab: 1128, Pembrolizumab: 1459, Atezolimab: 751) (Table 1).
Among the eleven studies, nine involved PD-1 antibodies (nivolumab: 5, pembrolizumab: 4) [4-12], and the other two involved PD-L1 antibodies (atezolizumab) [13, 14]. Additionally, three studies had data from melanoma(MM) patients [4-6], six from non-small cell lung cancer (NSCLC) patients $[7-10,13,14]$, one from urothelial cancer [11] and one from head-neck squamous cell carcinoma (HNSCC) [12] Two have three-arm trials, in which two dosage pembrolizumab arms were compared with the chemotherapy treatment $[6,10]$.

The Cochrane risk of bias tool was used to measure the quality of the included studies and the results are shown in Supplementary Figure S2.

\section{Analysis of colitis risk}

\section{Comparison of different PD-1/PD-L1 inhibitors treatments with chemotherapy}

As shown in Figure 1A, when compared with chemotherapy, there was a significant increase in the risk of grade 1-5 colitis incidence for PD-1 inhibitor (RR 2.69, 95\% CI: 1.15-6.29, p=0.023). However, there was no significant increase in the risk of grade 1-5 colitis incidence for PD-L1 inhibitor (atezolizumab) (RR 4.75, 95\% CI: 0.56-40.50, p=0.154). For high-grade colitis, the pooled RR of grade 3-5 colitis incidence was not significant for the PD-1 inhibitor subgroup (RR 2.53, 95\% CI: 0.96-6.68, p=0.060).

Table 1: Characteristics of the eligible RCTs

\begin{tabular}{|c|c|c|c|c|c|c|c|c|c|}
\hline Study[year] & Study type & Histology & Endpiont & Treatment arms & patients & $\begin{array}{l}\text { diarrhea } \\
\text { (G1-5) }\end{array}$ & $\begin{array}{l}\text { diarrhea } \\
\text { (G3-5) }\end{array}$ & $\begin{array}{l}\text { colitis } \\
\text { (G1-5) }\end{array}$ & $\begin{array}{l}\text { colitis } \\
\text { (G3-5) }\end{array}$ \\
\hline \multirow[t]{2}{*}{ weber[2015] } & III & MM & ORR & nivolumab 3mg/kg q2w & 268 & 30 & 1 & 3 & 2 \\
\hline & & & & Chemotherapy control & 102 & 15 & 2 & 0 & 0 \\
\hline \multirow[t]{2}{*}{$\operatorname{Reck}[2016]$} & III & NSCLC & OS & pembrolizumab 200mg q3w & 154 & 22 & 6 & 3 & 2 \\
\hline & & & & platinum-based chemotherapy & 150 & 20 & 2 & 0 & 0 \\
\hline \multirow[t]{2}{*}{ Robert[2015] } & III & MM & OS & nivolumab 3mg/kg q2w & 206 & 33 & 2 & 2 & 1 \\
\hline & & & & dacarbazine $(1000 \mathrm{mg} / \mathrm{m} 2) \mathrm{q} 3 \mathrm{w}$ & 205 & 32 & 1 & 0 & 0 \\
\hline \multirow[t]{2}{*}{ Ribas [2015]1 } & II & MM & ORR & pembrolizumab 2mg/kg q2w & 178 & 15 & 0 & 2 & 0 \\
\hline & & & & Chemotherapy control & 171 & 14 & 3 & 1 & 1 \\
\hline \multirow[t]{2}{*}{ Ribas[2015]2 } & II & MM & ORR & pembrolizumab $10 \mathrm{mg} / \mathrm{kg} \mathrm{q} 2 \mathrm{w}$ & 179 & 19 & 2 & 3 & 2 \\
\hline & & & & Chemotherapy control & 171 & 14 & 3 & 1 & 1 \\
\hline \multirow[t]{2}{*}{ Borghaei[2015] } & RCT III & NSCLC & OS & nivolumab 3mg/kg q2w & 287 & 22 & 2 & 2 & 1 \\
\hline & & & & DOX $75 \mathrm{mg} / \mathrm{m} 2 \mathrm{q} 3 \mathrm{w}$ & 268 & 62 & 3 & 0 & 0 \\
\hline \multirow[t]{2}{*}{ Brahmer[2015] } & RCT III & NSCLC & OS & nivolumab 3mg/kg q2w & 131 & 10 & 0 & 1 & 1 \\
\hline & & & & DOX 75mg/m2 q3w & 129 & 26 & 3 & 0 & 0 \\
\hline \multirow[t]{2}{*}{ Fehrenbacher[2016] } & RCT II & NSCLC & OS & atezolizumab $1200 \mathrm{mg} \mathrm{q} 3 \mathrm{w}$ & 142 & 10 & 1 & 2 & 1 \\
\hline & & & & DOX $75 \mathrm{mg} / \mathrm{m} 2 \mathrm{q} 3 \mathrm{w}$ & 135 & 30 & 3 & 0 & 0 \\
\hline \multirow[t]{2}{*}{ Herbst [2015]1 } & RCT III & NSCLC & OS & pembrolizumab $2 \mathrm{mg} / \mathrm{kg}$ q2w & 339 & 24 & 2 & 4 & 3 \\
\hline & & & & $\mathrm{DOX} 75 \mathrm{mg} / \mathrm{m} 2 \mathrm{q} 3 \mathrm{w}$ & 309 & 56 & 7 & 0 & 0 \\
\hline \multirow[t]{2}{*}{ Herbst [2015]2 } & RCT III & NSCLC & OS & pembrolizumab $10 \mathrm{mg} / \mathrm{kg} \mathrm{q} 2 \mathrm{w}$ & 343 & 22 & 0 & 2 & 1 \\
\hline & & & & $\mathrm{DOX} 75 \mathrm{mg} / \mathrm{m} 2 \mathrm{q} 3 \mathrm{w}$ & 309 & 56 & 7 & 0 & 0 \\
\hline \multirow[t]{2}{*}{ Rittmeyer [2017] } & RCT II & NSCLC & OS & atezolizumab $1200 \mathrm{mg} \mathrm{q} 3 \mathrm{w}$ & 609 & 98 & 4 & 2 & 0 \\
\hline & & & & DOX $75 \mathrm{mg} / \mathrm{m} 2 \mathrm{q} 3 \mathrm{w}$ & 578 & 141 & 11 & 0 & 0 \\
\hline \multirow[t]{2}{*}{ Bellmunt[2017] } & RCT III & Urothelial Ca & OS PFS & pembrolizumab 200mg q3w & 266 & 24 & 3 & 6 & 3 \\
\hline & & & & chemotherapy control & 255 & 33 & 2 & 1 & 0 \\
\hline \multirow[t]{2}{*}{ Ferris[2016] } & RCT III & head neck & OS & nivolumab 3mg/kg q2w & 236 & 16 & 0 & 0 & 0 \\
\hline & & & & standard therapy control & 111 & 15 & 2 & 1 & 0 \\
\hline
\end{tabular}




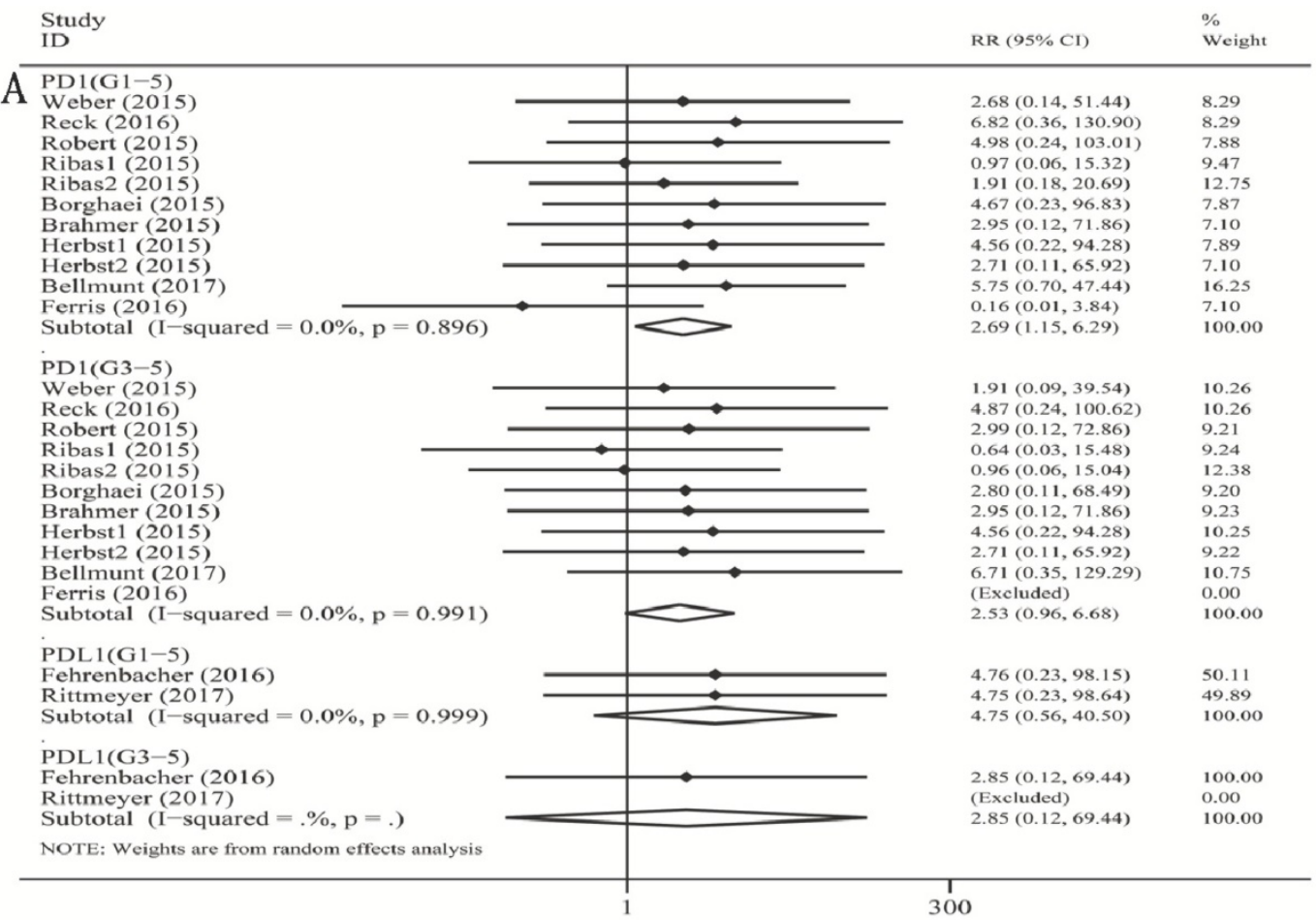

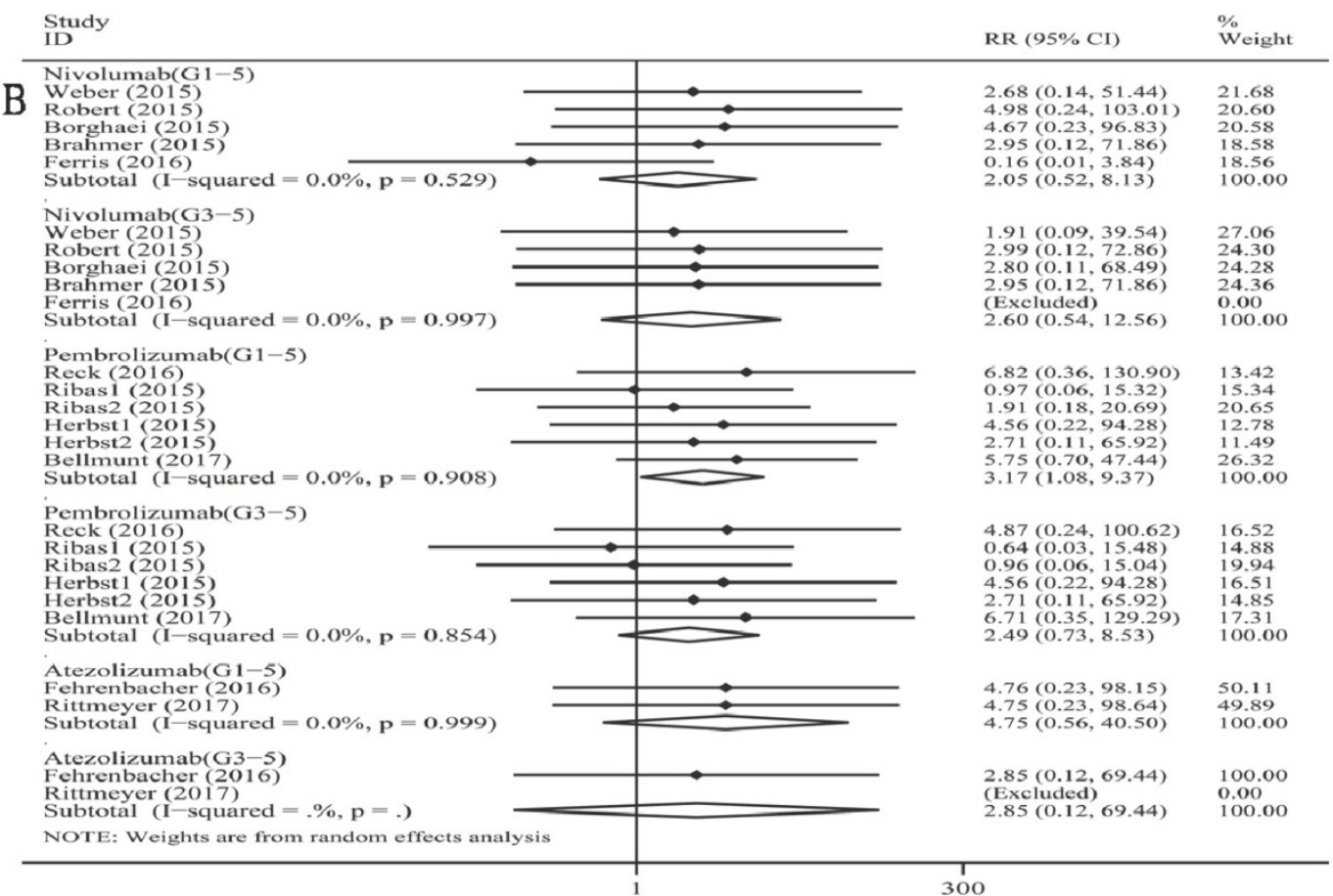

Figure 1: Forest plot analysis for colitis with PD-1/PD-L1 inhibitors versus chemotherapy. PD-1: PD-1 inhibitor V.S. chemotherapy; PD-L1: PD-L1 inhibitor V.S. chemotherapy. 1-5: grade1-5; 3-5: grade3-5. Nivolumab: nivolumab V.S. chemotherapy; Pembrolizumab: pembrolizumab V.S. chemotherapy; Atezolizumab: atezolizumab V.S. chemotherapy; G1-5: gradel-5; G3-5: grade3-5.

When compared with chemotherapy (Figure 1B), there was a significant increase in the risk of grade 1-5 colitis incidence for pembrolizumab (RR 3.17, 95\% CI: 1.08-9.37, $\mathrm{p}=0.037$ ). There was no significant increase in the risk of grade 1-5 colitis incidence for nivolumab (RR 2.05, 95\% CI: 0.52-8.13, $\mathrm{p}=0.305$ ), and for atezolizumab (RR 4.75, 95\% CI: 0.56-40.50, $\mathrm{p}=0.154$ ).
For high-grade colitis, the pooled RR of grade 3-5 colitis incidence was not significant for both nivolumab and pembrolizumab subgroup versus chemotherapy respectively (nivolumab: RR 2.60, 95\% CI: 0.54-12.56, $p=0.234$; pembrolizumab: RR $2.49,95 \%$ CI: 0.73-8.53, $\mathrm{p}=0.520$ ). 


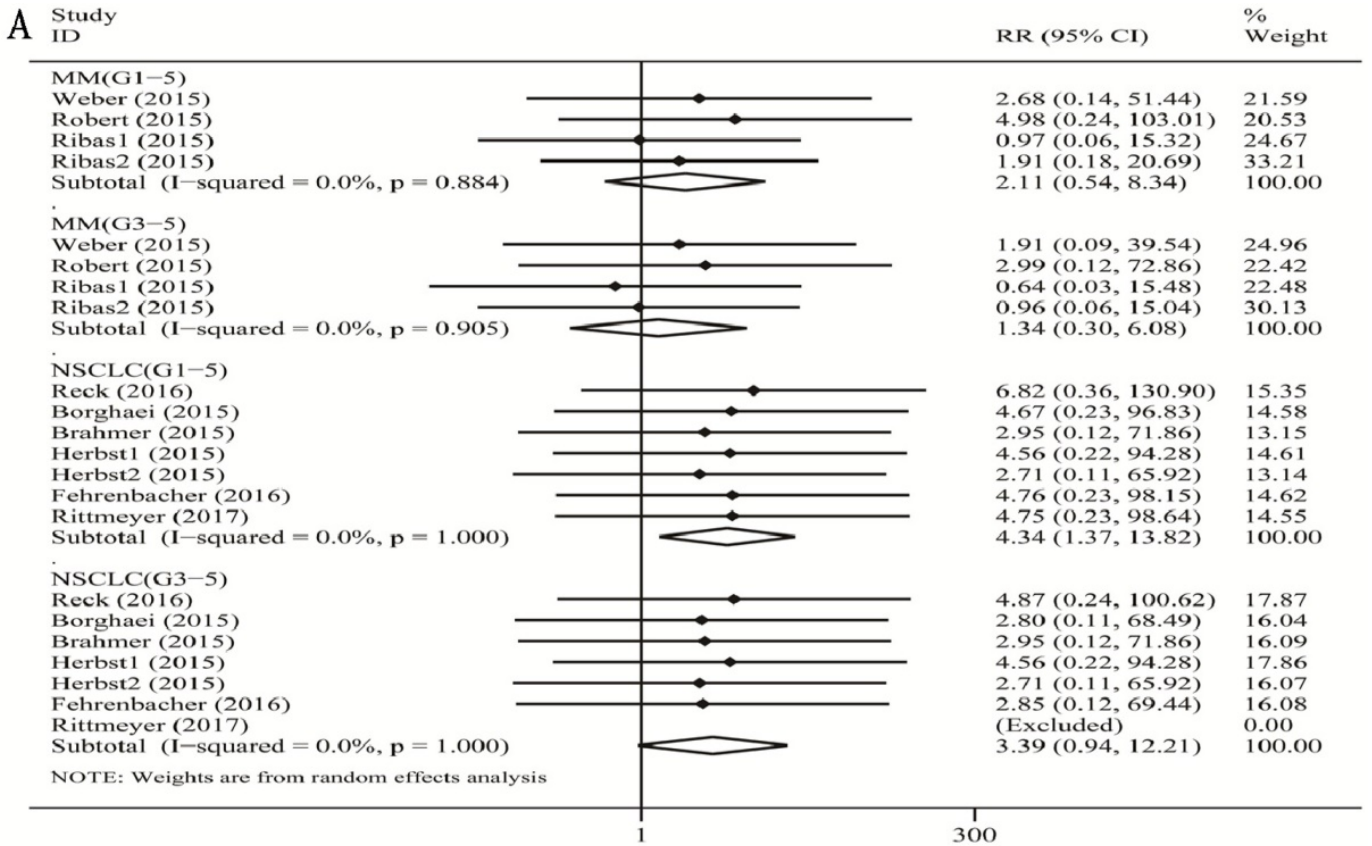

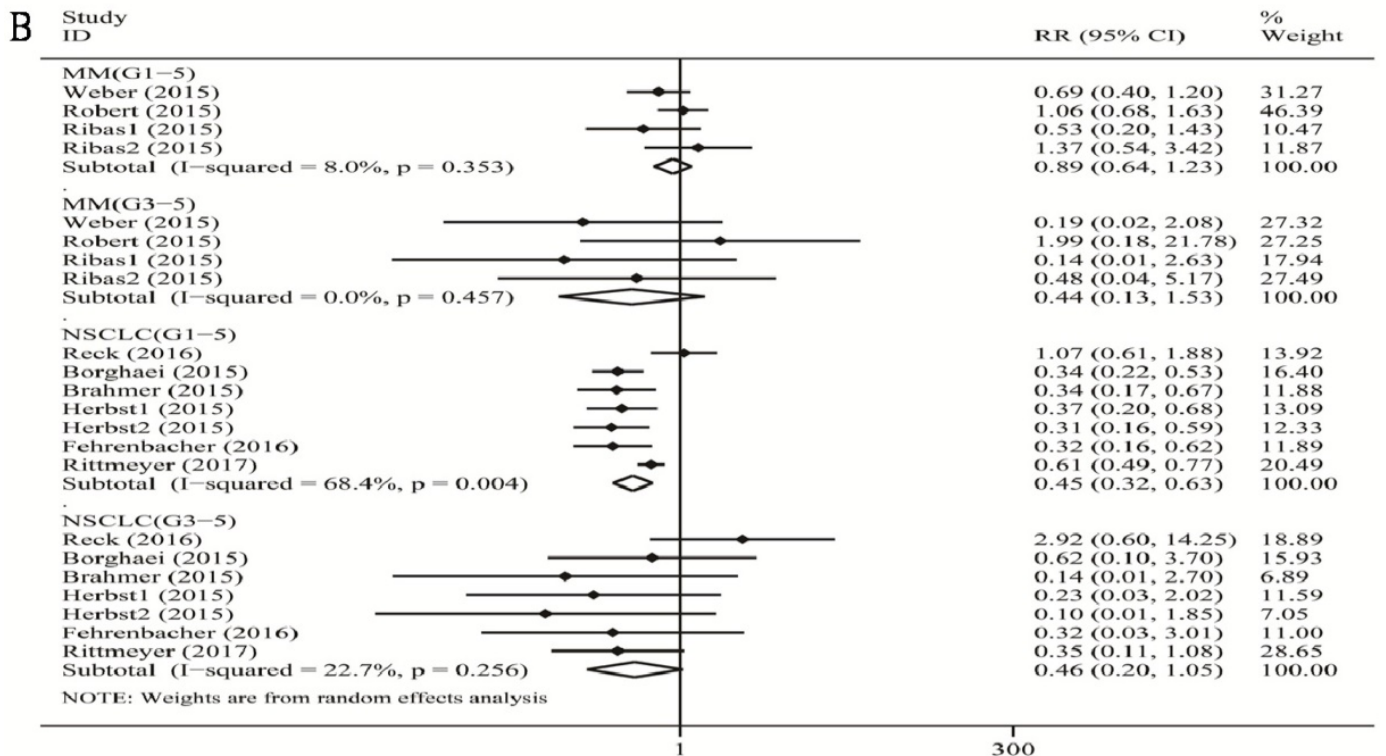

Figure 2: Forest plot analysis for colitis (A) and diarrhea (B) in different cancers with PD-1/PD-LI antibodies. MM: melanoma; Prostate: prostate cancer; NSCLC: non-small cell lung cancer; G1-5: grade1-5; G3-5: grade3-5.

We found a tiny overall heterogeneity in grade 1-5 colitis incidence. The PD-1 inhibitor group, the PD-L1 group (atezolizumab), the nivolumab subgroup, and the pembrolizumab subgroup all displayed $\mathrm{I}^{2}$ values of $0 \%$ (PD-1: $\mathrm{p}=0.896$; PD-L1 (atezolizumab): $\mathrm{p}=0.999 ;$ nivolumab: $\mathrm{p}=0.529$; pembrolizumab: $\mathrm{p}=0.908$ ). The tiny heterogeneity was also observed for grade 3-5 colitis incidence. The PD-1 inhibitor group, the nivolumab subgroup, and the pembrolizumab subgroup displayed $\mathrm{I}^{2}$ values of $0.0 \%$ (PD-1: $p=0.991$; nivolumab: $p=0.997$; pembrolizumab: $\mathrm{p}=0.854)$.
Comparison of PD-1/PD-L1 inhibitors treatment with chemotherapy for different cancer groups

When compared with chemotherapy (Figure 2A), there was a significant increase in the risk of grade 1-5 colitis incidence for PD-1/PD-L1 inhibitor treatment in patients with NSCLC (RR 4.34, 95\% CI: 1.37-13.82, $\mathrm{p}=0.013)$, and no significant increase in the risk of grade 1-5 colitis incidence for PD-1 inhibitor treatment in patients with MM (RR 2.11, 95\% CI: 0.54-8.34, $\mathrm{p}=0.285$ ). Additionally, there was no significant increase in the risk of grade 3-5 colitis incidence for PD-1 inhibitor treatment both with MM (RR 1.34, 95\% CI: 0.30-6.08, $p=0.705$ ), and for 
PD-1/PD-L1 inhibitor treatment with NSCLC (RR 3.39, 95\% CI: 0.94-12.21, $\mathrm{p}=0.061$ ).

We found a tiny overall heterogeneity in grade 1-5 colitis incidence. Both the MM patient subgroup and the NSCLC patient subgroup displayed $\mathrm{I}^{2}$ values of $0.0 \%$ (MM: $p=0.884$; NSCLC: $p=1.000$ ). The tiny heterogeneity was also observed for grade 3-5 colitis incidence in the MM subgroup and the NSCLC subgroup (MM: $\mathrm{I}^{2}=0 \%, \mathrm{p}=0.905$; NSCLC: $\mathrm{I}^{2}=0 \%, \mathrm{p}$ $=1.000$ ).

\section{Analysis of diarrhea risk}

\section{Comparison of different PD-1/PD-L1 inhibitors treatments with chemotherapy}

As shown in Figure 3A, when compared with chemotherapy, there was a significant decrease in the risk of grade 1-5 diarrhea incidence for PD-1 inhibitor treatment (RR 0.61, 95\% CI: 0.44-0.83, p=0.002), and a significant decrease in the risk of grade 1-5 diarrhea incidence for PD-L1 (atezolizumab) (RR 0.48, 95\% CI: $0.25-0.89, p=0.021)$ inhibitor treatment. When we analyzed high-grade diarrhea, we found that the pooled RR of grade 3-5 diarrhea incidence was significant for the PD-L1 inhibitor (atezolizumab) subgroup (RR 0.34, 95\% CI: 0.12-0.94, p=0.037).

When compared with chemotherapy (Figure 3B), we observed a significant decrease in the risk of grade 1-5 diarrhea incidence for nivolumab (RR 0.54, 95\% CI: 0.34-0.87, $\mathrm{p}=0.012$ ), and for atezolizumab (RR 0.48, 95\% CI: $0.25-0.89, \mathrm{p}=0.021)$. There was no significant decrease in the risk of grade 1-5 diarrhea incidence for pembrolizumab (RR 0.67, 95\% CI: 0.42-1.08, p=0.097). For high-grade diarrhea, the pooled RR of grade 3-5 diarrhea incidence was significant for atezolizumab subgroup (RR 0.34, 95\% CI: 0.12-0.94, p=0.037), not significant for both nivolumab and pembrolizumab subgroup respectively (nivolumab: RR $0.41,95 \% \mathrm{CI}$ : 0.14-1.17, $\mathrm{p}=0.095$; pembrolizumab: RR 0.56, 95\% CI: $0.19-1.70, \mathrm{p}=0.308)$.

There was a substantial overall heterogeneity of grade 1-5 diarrhea incidence in all the subgroups including PD-1 inhibitor subgroup, the PD-L1 subgroup (atezolizumab), the nivolumab subgroup, and the pembrolizumab subgroup (PD-1: $\mathrm{I}^{2}=74.7 \%$, $\mathrm{p}$ $=0.000$; nivolumab: $\mathrm{I}^{2}=74.6 \%, \quad \mathrm{p}=0.003$; pembrolizumab: $\mathrm{I}^{2}=78.8 \%, \mathrm{p}=0.001$; atezolizumab: $\mathrm{I}^{2}=69.6 \%, \mathrm{p}=0.070$ ). For grade 3-5 diarrhea incidence, the heterogeneity was tiny for the PD-L1 inhibitor (atezolizumab) subgroup and nivolumab subgroup (atezolizumab: $\mathrm{I}^{2}=0 \%, \mathrm{p}=0.947$; nivolumab: $\mathrm{I}^{2}=0 \%, \mathrm{p}$ $=0.448)$, low for PD-1 subgroup $\left(\mathrm{I}^{2}=26.8 \%, \mathrm{p}=0.189\right)$, and moderate for the pembrolizumab subgroup $\left(\mathrm{I}^{2}=47.6 \%, \mathrm{p}=0.080\right)$.
As shown in Supplementary Table S4, the sensitivity analysis was used to examine whether the results could have been influenced for PD-1 (Grade1-5 diarrhea) subgroup, nivolumab (Grade1-5 diarrhea) subgroup, and pembrolizumab (Grade 1-5 diarrhea) subgroup respectively.

\section{Comparison of PD-1/PD-L1 inhibitor treatment with chemotherapy for different cancer groups}

When compared with chemotherapy (Figure 2B), we observed a significant decrease in the risk of grade 1-5 diarrhea incidence for PD-1/PD-L1 inhibitor in patients with NSCLC (RR 0.45, 95\% CI: 0.32-0. 63, $\mathrm{p}<0.001$ ), but no significant decrease in the risk of grade 1-5 diarrhea incidence for PD-1 inhibitor in patients with MM (RR 0.89, 95\% CI: 0.64-1.23, $\mathrm{p}=0.457)$. However, there was no significant decrease in the risk of grade 3-5 colitis incidence with PD-1/PD-L1 inhibitor with NSCLC (RR 0.46, 95\% CI: 0.20-1.05, $\mathrm{p}=0.065$ ), and with MM (RR 0.44, 95\% CI: $0.13-1.53, \mathrm{p}=0.196)$.

We found a substantial overall heterogeneity of grade 1-5 diarrhea incidence in the NSCLC subgroup $\left(\mathrm{I}^{2}=68.4 \%, \mathrm{p}=0.004\right)$, but a low heterogeneity in the MM subgroup $\left(I^{2}=8.0 \%, p=0.353\right)$. For grade $3-5$ diarrhea incidence, the heterogeneity was tiny for the MM subgroup $\left(\mathrm{I}^{2}=0 \%, \mathrm{p}=0.457\right)$, and low for the NSCLC subgroup (MM: $\mathrm{I}^{2}=22.7 \%, \mathrm{p}=0.256$ ).

As shown in Supplementary Table S4, the sensitivity analysis was used to examine whether the results could have been influenced for NSCLC subgroup (Grade1-5 diarrhea).

\section{Analysis of publication bias}

Egger's test and Begg's test, conducted in STATA 12.0 software, were used to evaluate the publication bias between different RCTs. As presented in Supplementary Table S3 and Supplementary Figure S3, all the P values were $>0.05$ after both tests. Therefore, there was no significant publication bias in this meta-analysis.

\section{Discussion}

Although one previous meta-analysis showed that ipilimumab and overall PD-1/PD-L1 inhibitors (PD-1 lumped with PD-L1 inhibitors) were associated with the increase of colitis incidence [21], but not with each PD-1 and PD-L1 inhibitor respectively, such as nivolumab, pembrolizumab and atezolizumab. As far as we know, this is the first systematic review and meta-analysis on the risk of immune-related colitis following the use of nivolumab, pembrolizumab and atezolizumab in patients with advanced solid tumors. 
A $\underset{\text { ID }}{\text { Study }}$

RR $(95 \%$ CI) $\quad$ Weight

PD1(G1-5)

Weber (2015)

Reck (2016)

Ribas1 (2015)

Ribas2 (2015)

Brahmer (2015)

Herbst2 (2015)

Bellmunt (2017)

Ferris (2016)

Subtotal (I-squared $=74.7 \%, p=0.000$ )

PD1(G3-5)

Weber (2015)

Reck (2016)

Ribas 1 (2015)

Ribas2 (2015)

Borghaei (2015)

Brahmer (2015)

Herbst 1 (2015)

Herbst2 (2015)

Bellmunt (2017)

Subtotal $(\mathrm{I}-$-squared $=26.8 \%, \mathrm{p}=0.189)$

PDL 1(G1-5)

Fehrenbacher (2016)

Rittmeyer (2017)
Subtotal (I-squared $=69.6 \%, p=0.070$ )

PDL 1(G3-5)

Fehrenbacher (2016)

Subtotal (1-squared $=0.0 \%, p=0.947)$

NOTE: Weights are from random effects analysis

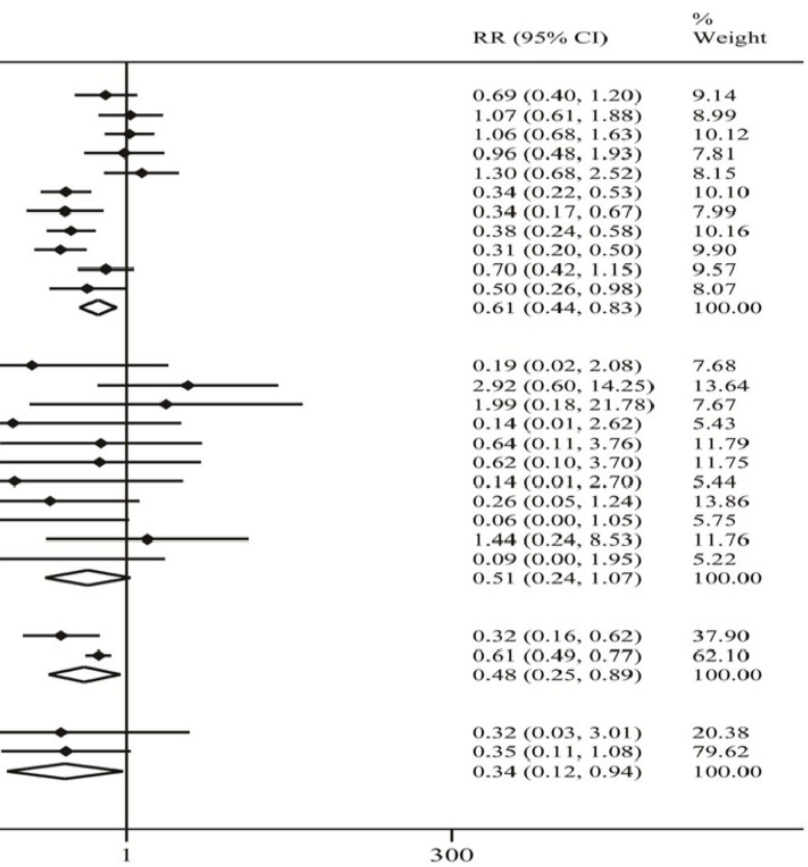

B

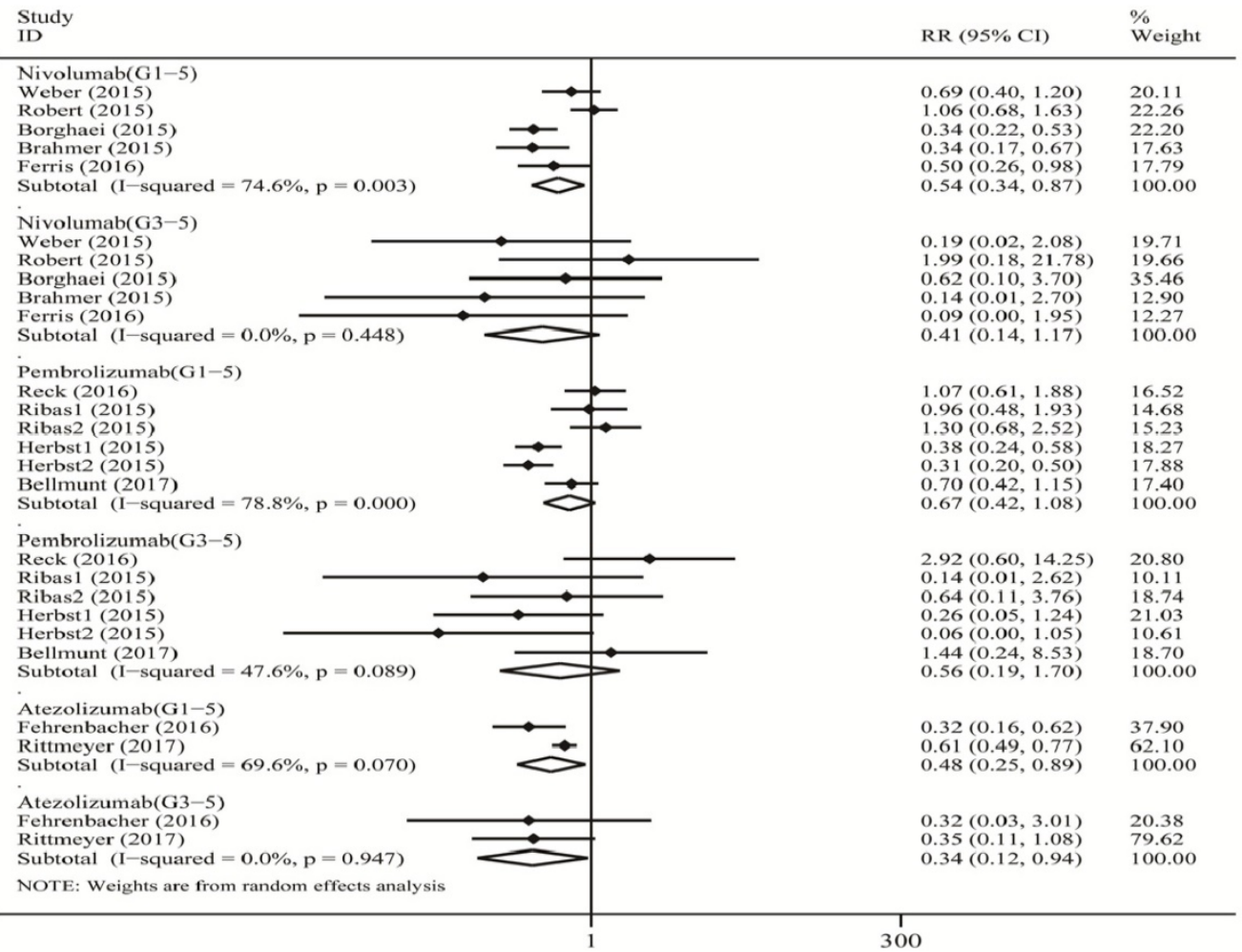

Figure 3: Forest plot analysis for diarrhea with PD-1/PD-L1 inhibitors versus chemotherapy. PD-1: PD-1 inhibitor V.S. chemotherapy; PD-LI: PD-L1 inhibitor V.S. chemotherapy. 1-5: grade1-5; 3-5: grade3-5.MM: melanoma; NSCLC: non-small cell lung cancer; G1-5: grade1-5; G3-5: grade3-5.

Our meta-analysis includes thousands of patients (Nivolumab: 1128, Pembrolizumab: 1459, Atezolimab: 751). It shows that pembrolizumab increases the risk of all-grade colitis incidence (about three times), but not the risk of high-grade colitis incidence and that of all-grade / high-grade diarrhea incidence when compared with chemotherapy. In addition, PD-L1 antibody (atezolizumab) may reduce the risk of all-grade diarrhea incidence, and furthermore, reduce the risk of grade 3-5 diarrhea incidence. However, nivolumab does not increase the risk of all/high-grade colitis incidence but reduces the risk of only all-grade diarrhea incidence (about one half), when compared with chemotherapy. The risks of colitis and diarrhea are different in different types of solid cancers with PD-1/PD-L1 antibodies (MM 
and NSCLC). PD-1/PD-L1 inhibitor treatment in patients with NSCLC increases the risk of all-grade colitis incidence (about four times), but not that of high-grade colitis incidence, and decreases the risk of all-grade diarrhea incidence (about four tenths). However, PD-1/PD-L1 inhibitor treatment in patients with MM does not change the risk of all-grade/high-grade colitis and diarrhea incidence.

Although our results confirm that PD-1 inhibitors tend to increase the risk of immune-induced colitis, and the mechanism underlying its emergence is largely unknown. A byproduct of the anti-tumor function of the PD-1 inhibitor is to inhibit the binding of PD-1 and its ligand PD-L1/2, which blocks the reception of prohibitive signals by $\mathrm{T}$ cells to enable them to activate immune responses against tumor cells. The function of PD-1 inhibitors may also disturb the balance of autologous tolerance [22]. For instance, the PD-1 inhibitors may liberate $\mathrm{T}$ cells' reactivity to healthy tissues and thus lead to the production of autoantibodies, the release of excessive inflammatory factors (e.g. IFNa, TNF, IL-23/IL-17 inflammatory axis) [23-26], and NETosis, which cause harm to healthy cells [27]. Clinical reports state that the incidence of colitis with PD-1/PD-L1 antibodies is much lower than with CTLA4 antibodies [28, 29]. Consistently, the morbidity of colitis after PD-1 inhibitor treatment $(0.6 \%-2.3 \%)$ seems higher than that after PD-L1 inhibitor treatment (0.3\%-1.4\%). These morbidity values were calculated based on the literature included in our meta-analysis [4-14].

Our meta-analysis reveals that PD-1/PD-L1 antibodies reduce the risk of diarrhea when compared with chemotherapy, and PD-L1 antibodies even reduce the risk of severe diarrhea. This may be attributed to the fact that chemotherapy-induced diarrhea is a very common problem in cancer patients, with an incidence as high as 50\%-80\% [30]. In contrast, PD-1 antibodies increase the risk of colitis when compared with chemotherapy. These observations support the idea that PD-1 antibodies introduce a larger risk of immune-related colitis that cannot be determined solely by diarrhea, one of its common symptoms. We failed to observe a significant increase in the risk of colitis for PD-L1 antibodies when compared with chemotherapy, which is possibly due to the current scarcity of RCTs involving PD-L1 inhibitors. Another reason may be that PD-L1 antibodies only block binding to PD-L1, but not PD-L2, and so treatment with PD-L1 antibodies may be accompanied by less immune-related toxicity than PD-1 antibodies in part by its selective modulation of the immune response [31]. One study showed that after the PD-L1-PD-1 pathway, the PD-L2-PD-1 pathway is second in regulating $\mathrm{T}$ cell responses [32]. In the same way, our previously published study also indicated that, when compared with chemotherapy, PD-1/PD-L1 antibody treatment has beneficial effects on advanced NSCLC patient monotherapy, along with fewer conventional chemotherapy adverse events such as diarrhea and fever, but increased immune-associated adverse effects [33].

The type of meta-analysis itself based on published data unavoidably had several limitations [34]. The first limitation was that we had no individual patient data, which would have provided more detail about immune-related colitis with PD-1/PD-L1 inhibitors. Secondly, we assume that the diagnostic criteria of immune-related colitis were consolidated and identical for every RCT, but that of diarrhea was not, because the diagnosis of diarrhea was subjective both for patients and clinicians. So, the considerable heterogeneity of diarrhea could be explained partially. Thirdly, there are still some questions we cannot explain well, such as that both nivolumab and pembrolizumab belong to PD-1 inhibitors, but only pembrolizumab in our meta-analysis cause more risk of immune-related colitis than chemotherapy. Recently one study has shown that pembrolizumab and nivolumab have no overlapping binding areas on PD-1 with each other. Those differences in PD-1 binding sites might be responsible for the difference in a risk of immune-mediated colitis between pembrolizumab and nivolumab [35]. On the other hand, we have made great efforts on the overall quality assessment that maybe make our conclusion more balanced and credible: (1) two independent reviewers searched all the relevant trails with well-defined inclusion criteria. They assessed studies appropriate for meta-analysis evaluated, and assessed the risk of bias for the included RCTs. (2) Two independent reviewers verified data in our meta-analysis which was performed by pair-wise comparisons. (3) The REM, subgroup analysis, and sensitivity analysis were employed statistically.

Overall, our meta-analysis reminds physicians of the possibility of immune-induced colitis in cancer patients treated with PD-1 inhibitor (especially pembrolizumab), particularly in those with symptoms of diarrhea that is severe or cannot be cured by routine treatment. Serious colitis may be fatal, which renders its symptoms worthwhile monitoring in order to avoid neglecting this risk. Further study on the molecular mechanisms underlying PD-1 inhibitorrelated colitis could help us to prevent or relieve this side effect during treatment [36]. 


\section{Conclusion}

In summary, our meta-analysis demonstrates that PD-1 inhibitor may result in a higher risk of all-grade immune-induced colitis than chemotherapy. Pembrolizumab may result in a higher risk of all-grade immune-mediated colitis than chemotherapy. However, both nivolumab and atezolizumab maybe do not introduce a higher risk of immunemediated colitis than chemotherapy. The PD1/PD-L1 inhibitor may increase the risk of grade 1-5 colitis incidence than chemotherapy in patients with NSCLC, but not in patients with MM.

\section{Supplementary Material}

Supplementary figures and tables. http://www.jcancer.org/v09p1614s1.pdf

\section{Acknowledgments}

This work was supported by the Foundation of clinical research of Capital Medical University (No.15JL33) and the Foundation of Beijing friendship hospital (yyqdkt 2014-12).

\section{Author Contributions}

Q.S., X.H.S., and Y.L.H. had access to all the data included in the study and are responsible for the completeness of the data and the accuracy of our analysis. Y.L.H. helped to design the study, Q.S., and X.C.Z. contributed to the statistical analysis and the revision of this manuscript. Q.S., Z.H.G. and Z.G.S. approved the final manuscript.

\section{Competing Interests}

The authors have declared that no competing interest exists.

\section{References}

1. Siegel RL, Miller KD, Jemal A. Cancer Statistics, 2017. CA Cancer J Clin 2017; 67:7-30.

2. Sharma P, Allison JP. The future of immune checkpoint therapy. Science 2015; 348:56-61.

3. Johnson DB, Sullivan RJ, Menzies AM. Immune checkpoint inhibitors in challenging populations. Cancer 2017; 123:1904-1911.

4. Weber JS, D'Angelo SP, Minor D et al. Nivolumab versus chemotherapy in patients with advanced melanoma who progressed after anti-CTLA-4 treatment (CheckMate 037): a randomised, controlled, open-label, phase 3 trial. Lancet Oncol 2015; 16:375-384.

5. Robert C, Long GV, Brady B et al. Nivolumab in previously untreated melanoma without BRAF mutation. N Engl J Med 2015 372:320-330.

6. Ribas A, Puzanov I, Dummer $R$ et al. Pembrolizumab versus investigator-choice chemotherapy for ipilimumab-refractory melanoma (KEYNOTE-002): a randomised, controlled, phase 2 trial. Lancet Oncol 2015; 16:908-918.

7. Reck M, Rodríguez-Abreu D, Robinson AG et al. Pembrolizumab versus Chemotherapy for PD-L1-Positive Non-Small-Cell Lung Cancer. N Engl J Med 2016; 375:1823-1833.

8. Borghaei H, Paz-Ares L, Horn L et al. Nivolumab versus Docetaxel in Advanced Nonsquamous Non-Small-Cell Lung Cancer. N Engl J Med 2015; 373:1627-1639.

9. Brahmer J, Reckamp KL, Baas P et al. Nivolumab versus Docetaxel in Advanced Squamous-Cell Non-Small-Cell Lung Cancer. N Engl J Med 2015; 373:123-135.
10. Herbst RS, Baas P, Kim DW et al. Pembrolizumab versus docetaxel for previously treated, PD-L1-positive, advanced non-small-cell lung cancer (KEYNOTE-010): a randomised controlled trial. Lancet 2016; 387:1540-1550.

11. Bellmunt J, de Wit R, Vaughn DJ et al. Pembrolizumab as Second-Line Therapy for Advanced Urothelial Carcinoma. N Engl J Med 2017; 376:1015-1026.

12. Ferris RL, Blumenschein G, Fayette J et al. Nivolumab for Recurrent Squamous-Cell Carcinoma of the Head and Neck. N Engl J Med 2016; 375:1856-1867

13. Fehrenbacher L, Spira A, Ballinger $\mathrm{M}$ et al. Atezolizumab versus docetaxel for patients with previously treated non-small-cell lung cancer (POPLAR): a multicentre, open-label, phase 2 randomised controlled trial. Lancet 2016; 387:1837-1846.

14. Rittmeyer A, Barlesi F, Waterkamp D et al. Atezolizumab versus docetaxel in patients with previously treated non-small-cell lung cancer (OAK): a phase 3, open-label, multicentre randomised controlled trial. Lancet 2017; 389:255-265.

15. Verschuren EC, van den Eertwegh AJ, Wonders I, Slangen RM, van Delft F, van Bodegraven A, Neefjes-Borst A, de Boer NK. Clinical, Endoscopic, and Histologic Characteristics of Ipilimumab-Associated Colitis. Clin Gastroenterol Hepatol 2016; 14:836-842.

16. Higgins JPT, Green S. Cochrane Handbook for Systematic Reviews of Interventions Version 5.1.0. The Cochrane Collaboration, 2011. Available at: http://www.cochrane-handbook.org.

17. Moher D, Liberati A, Tetzlaff J, Altman DG; The PRISMA Group. Preferred reporting items for systematic reviews and meta-analyses: the PRISMA statement. PLoS Med 2009; 6: e1000097.

18. Huang X, Lin J, Demner-Fushman D. Evaluation of PICO as a knowledge representation for clinical questions. AMIA Annu Symp Proc 2006: 359-363.

19. Higgins JP, Thompson SG, Deeks JJ, Altman DG. Measuring inconsistency in meta-analyses. BMJ 2003; 327:557-560.

20. DerSimonian R, Laird N. Meta-analysis in clinical trials revisited. Contemp Clin Trials 2015; 45:139-145.

21. Wang DY, Ye F, Zhao S, Johnson DB. Incidence of immune checkpoint inhibitor-related colitis in solid tumor patients: A systematic review and meta-analysis. Oncoimmunology 2017; 6:e1344805.

22. Friedman CF, Proverbs-Singh TA, Postow MA. Treatment of the Immune-Related Adverse Effects of Immune Checkpoint Inhibitors: A Review. JAMA Oncol 2016; 2:1346-1353.

23. Coutzac C, Adam J, Soularue E et al. Colon Immune-Related Adverse Events: Anti-CTLA-4 and Anti-PD-1 Blockade Induce Distinct Immunopathological Entities. J Crohns Colitis 2017; 11:1238-1246.

24. Gaffen SL, Jain R, Garg AV, Cua DJ. The IL-23-IL-17 immune axis: from mechanisms to therapeutic testing. Nat Rev Immunol 2014; 14:585-600.

25. Lubberts E. The IL-23-IL-17 axis in inflammatory arthritis. Nat Rev Rheumatol 2015; 11:415-429.

26. Pauken KE, Sammons MA, Odorizzi PM et al. Epigenetic stability of exhausted T cells limits durability of reinvigoration by PD-1 blockade. Science 2016; 354:1160-1165.

27. van der Vlist M, Kuball J, Radstake TR, Meyaard L. Immune checkpoints and rheumatic diseases: what can cancer immunotherapy teach us. Nat Rev Rheumatol 2016; 12:593-604.

28. Hamid O, Robert $C$, Daud A et al. Safety and tumor responses with lambrolizumab (anti-PD-1) in melanoma. N Engl J Med 2013; 369:134-144.

29. Boutros C, Tarhini A, Routier E et al. Safety profiles of anti-CTLA-4 and anti-PD-1 antibodies alone and in combination. Nat Rev Clin Oncol 2016; 13:473-486.

30. Stein A, Voigt W, Jordan K. Chemotherapy-induced diarrhea: pathophysiology, frequency and guideline-based management. Ther Adv Med Oncol 2010; 2:51-63.

31. Philips GK, Atkins M. Therapeutic uses of anti-PD-1 and anti-PD-L1 antibodies. Int Immunol 2015; 27:39-46.

32. Latchman Y, Wood CR, Chernova T et al. PD-L2 is a second ligand for PD-1 and inhibits T cell activation. Nat Immunol 2001; 2:261-268.

33. Su Q, Sun Z, Zhang C, Hou Y, Cao B. PD-1/PD-L1 antibodies efficacy and safety versus docetaxel monotherapy in advanced NSCLC patients after first-line treatment option: systems assessment. Oncotarget 2017; 8:59677-59689

34. Lyman GH, Kuderer NM. The strengths and limitations of meta-analyses based on aggregate data. BMC Med Res Methodol 2005; 5:14.

35. Tan S, Zhang H, Chai Y, Song H, Tong Z, Wang Q, Qi J, Wong G, Zhu X, Liu WJ, Gao S, Wang Z, et al. An unexpected N-terminal loop in PD-1 dominates binding by nivolumab. Nat Commun 2017; 8:14369

36. Sznol M, Postow MA, Davies MI, Pavlick AC, Plimack ER, Shaheen M, Veloski C, Robert C. Endocrine-related adverse events associated with immune checkpoint blockade and expert insights on their management. Cancer Treat Rev 2017; 58:70-76. 